\title{
Lower use of dental services among long term cigarette smokers
}

\author{
L A Mucci, D R Brooks
}

\begin{abstract}
Study objective-Given the advanced stage of most oral cancer cases at diagnosis, it is hypothesised that a significant proportion of higher risk adults do not visit a dentist annually. The study objectives were to assess whether long term smokers were less likely to visit the dentist.

Design-Data from the 1998 Massachusetts Behavioral Risk Factor Surveillance System, a population-based, random digit dial telephone health survey, were used to evaluate whether adults at higher risk of oral cancer attributable to long term cigarette smoking were less likely to go to the dentist, controlling for socioeconomic, demographic, and health related characteristics.
\end{abstract}

Patients-A representative sample of 2119 Massachusetts adults aged 35 and older. Main results-Adults who were long term smokers were less likely than never smokers to have visited the dentist in the previous year (adjusted $O R=0.69,95 \%$ confidence intervals $(C I)=0.48,0.99)$. Moreover, adults who were at higher risk from both long term smoking and low fruit and vegetable consumption were even less likely to visit the dentist than adults with neither risk factor (adjusted OR $=0.39,95 \%$ CI $=0.22,0.68)$. Among long term smokers, the likelihood of a yearly examination decreased with increasing smoking duration and amount smoked per day.

Conclusions-These findings support the hypothesis that adults at higher risk of oral cancer attributable to long term cigarette smoking are less likely to have routine dental examinationss, even controlling for socioeconomic and health related differences.

(F Epidemiol Community Health 2001;55:389-393)

Massachusetts Department of Public Health, Bureau of Health Statistics, Research and Evaluation, 250 Washington Street, 6th floor, Boston, MA 02108, USA

Correspondence to: Dr Mucci

(lmucci@hsph.harvard.edu)

Accepted for publication 1 February 2001
The burden of suffering from oral cancer is largely preventable. Early diagnosis of preclinical oral cancer or precancer involves a simple, inexpensive examination of the oral cavity, and early treatment provides good prognosis for patients. Yet, survival from oral cancer is among the lowest of all cancer sites, primarily because of the advanced stage at diagnosis. ${ }^{1}$ Treatments for localised oral cancer yield five year survival rates as high as $86 \%$, compared with $42 \%$ for regional and $22 \%$ for distant disease. $^{23}$ Only $35 \%$ of patients in the US diagnosed with oral cancer are diagnosed at a localised stage. ${ }^{2}$
At least $75 \%$ of oral malignancies are preceded by a precancer stage, such as leucoplakia, at the site of cancer development. ${ }^{45}$ Within five years, $5 \%$ of precancers will transform to neoplasia. ${ }^{56}$ Early detection of oral precancer is important because treatment could lead to a reduction in oral cancer incidence. Clinical trials have demonstrated that chemotherapeutic treatment of leucoplakia leads to remission of precancer and prevention of oral cancer lesions. ${ }^{78}$

If precancerous lesions progress to malignancy, treatment of early stage cancers may still lead to reductions in morbidity and mortality. There is consistent evidence from observational data that persons with early stage oral cancer have better survival than those diagnosed with advanced disease. ${ }^{13}$ Earlier diagnosis could also decrease patient suffering because of disabilities and disfigurement from more aggressive cancer treatment. ${ }^{1}$

Epidemiological data suggest that precancerous lesions may persist for 5-10 years before becoming malignant, and that oral cancer exists in a preclinical, detectable stage (sojourn time) for approximately one year. ${ }^{910}$ Thus, yearly examinations by a dentist, including a simple inspection and palpation of the oral cavity, could identify disease at an earlier, more treatable stage. While all people should visit a dentist yearly, patients at higher risk of oral cancer should in particular be encouraged to see a dentist annually.

Tobacco is a component cause in at least $80 \%$ of oral cancers. ${ }^{112}$ People who use tobacco in the form of cigarettes are at 4-12 times increased risk of disease. ${ }^{13-15}$ As most cases of oral cancer are diagnosed at an advanced stage of disease, we hypothesise that a significant proportion of adults at higher risk are not seeing a dentist for yearly examinations. To explore this hypothesis, we used population based surveillance data to assess whether people at higher risk of oral cancer attributable to long term tobacco use were less likely to visit a dentist within the year.

\section{Methods}

The analyses in this paper are based on data collected as part of the Behavioral Risk Factor Surveillance System (BRFSS) in Massachusetts from 1 January to 31 December 1998. The BRFSS is an ongoing, random digit dial, telephone survey of adults age 18 and older, and is conducted in all states as a joint collaboration between the Centers for Disease Control and Prevention (CDC) and State Departments of Health. Telephone numbers are selected 
randomly within geographical strata, and multiple attempts are made to reach each household. To be eligible to participate in the survey, the household had to be occupied by at least one adult aged 18 and older. Institutions, group quarters of 10 or more unrelated adults, and temporary residences, such as summer homes, were excluded. Once an eligible household is contacted, one adult from the household is randomly selected for interview. No proxy respondents were allowed for any reason, including language barrier, disability or lack of availability. The data are then weighted by the probability of selection, which is related to the number of adults and telephone lines in the household and, in Massachusetts, town of residence, as well as differential participation by sex, age, and race. Characteristics of the BRFSS are described in detail elsewhere. ${ }^{16}$

Trained interviewers collected data on a variety of health characteristics, risk factors for chronic conditions, and preventive behaviours. In 1998, the Massachusetts BRFSS also included a CDC developed module on oral health, with questions related to time since last dental visit, reasons for not visiting a dentist within the preceding 12 months, dental insurance, and the number of teeth lost to decay. Respondents also provided demographic information and answered questions on diverse topics such as smoking, fruit and vegetable consumption, and healthcare access and utilisation.

We restricted our analyses to respondents age 35 and older, based on the higher incidence of oral cancer among this age group. We classified respondents on cigarette use based on epidemiological evidence. Several studies have shown a dose-response relation between both

Table 1 Percentage of adults age 35 and older reporting recent dental visit, by smoking status and selected demographic and health characteristics, Massachusetts BRFSS, 1998

\begin{tabular}{|c|c|c|c|c|}
\hline & \multicolumn{4}{|c|}{ Recent dental visit (percentage and 95\% confidence intervals) } \\
\hline & \multicolumn{2}{|c|}{ Never smoker $(n=1473)$} & \multicolumn{2}{|c|}{ Long term smoker $(n=646)$} \\
\hline & $\%$ & $95 \% C I$ & $\%$ & $95 \% C I$ \\
\hline Overall & 83.0 & $80.5,85.6$ & 71.1 & $66.1,75.9$ \\
\hline \multicolumn{5}{|l|}{ Gender } \\
\hline male & 83.8 & $79.6,87.9$ & 67.7 & $60.0,75.4$ \\
\hline female & 82.5 & $79.3,85.7$ & 74.3 & $68.5,80.1$ \\
\hline \multicolumn{5}{|l|}{ Age group } \\
\hline $35-54$ & 87.7 & $84.6,90.7$ & 80.8 & $71.3,81.6$ \\
\hline $55-74$ & 85.2 & $77.0,86.6$ & 59.7 & $47.1,69.1$ \\
\hline 75 and older & 66.8 & $58.7,74.9$ & 10.4 & $0,25.2$ \\
\hline \multicolumn{5}{|l|}{ Education } \\
\hline$<$ high school grad & 47.8 & $35.4,60.2$ & 58.3 & $42.5,74.2$ \\
\hline high school grad & 76.1 & $70.6,81.5$ & 71.8 & $64.0,79.5$ \\
\hline college, $1-3$ yrs & 86.0 & $81.2,90.7$ & 70.6 & $62.0,79.2$ \\
\hline college, $4+$ yrs & 92.3 & $89.5,95.2$ & 82.4 & $74.5,90.4$ \\
\hline \multicolumn{5}{|l|}{ Household income } \\
\hline$<\$ 25000$ & 71.8 & $63.6,80.0$ & 57.0 & $43.7,70.4$ \\
\hline$\$ 25-49999$ & 73.1 & $65.0,81.3$ & 66.3 & $56.0,76.6$ \\
\hline$\$ 50-74999$ & 91.6 & $88.2,94.9$ & 74.5 & $67.2,81.7$ \\
\hline$\$ 75000$ or more & 90.9 & $86.1,95.8$ & 85.7 & $73.7,97.8$ \\
\hline \multicolumn{5}{|l|}{ Dental insurance } \\
\hline insured & 87.7 & $84.7,90.7$ & 82.9 & $78.2,87.6$ \\
\hline uninsured & 76.0 & $71.5,80.4$ & 52.2 & $43.4,61.1$ \\
\hline \multicolumn{5}{|l|}{ Health insurance } \\
\hline insured & 83.9 & $81.4,86.5$ & 73.8 & $68.8,78.8$ \\
\hline not insured & 65.4 & $50.1,80.8$ & 40.5 & $25.2,55.8$ \\
\hline \multicolumn{5}{|c|}{ Routine medical check up } \\
\hline yes & 84.4 & $81.7,87.1$ & 75.4 & $69.8,81.0$ \\
\hline no & 77.6 & $70.9,84.2$ & 61.2 & $51.5,70.9$ \\
\hline \multicolumn{5}{|c|}{ Teeth lost to decay/gum disease } \\
\hline none to 5 & 88.0 & $85.4,90.5$ & 77.2 & $71.5,82.9$ \\
\hline 6 or more & 65.9 & $58.4,73.4$ & 56.9 & $50.1,68.7$ \\
\hline
\end{tabular}

number of cigarettes and duration of smoking on increasing risk of oral cancer. ${ }^{14}{ }^{15}$ In addition, recent quitters seem to be at increased risk of disease for some years after quitting $^{14}{ }^{15}$ and are also at increased risk of recidivism. ${ }^{17}$ We categorised as long term smokers people who had smoked for at least 10 years and who were either current smokers or former smokers who quit smoking within the past year. We classified never smokers, defined as people who had smoked less than 100 cigarettes within their lifetime, as the referent group.

Our main outcome variable was self report of a recent dental visit within the year. People who had visited a dental office within the year were categorised as having had a recent dental visit, while people who visited the dentist more than one year ago or had never been to the dentist were categorised as not having a recent dental visit. This classification was based on the estimated sojourn time for oral cancer of one year. $^{9}{ }^{10}$

We assessed whether long term smokers were less likely to have had an annual dental visit relative to never smokers. To more fully understand the relation between cigarette smoking and use of dental services, we controlled for the following variables: age, education, gender, dental and health insurance, routine medical visit within the year, and number of permanent teeth lost to decay. Many of these variables have shown a positive association with tobacco, and may modify the relation between risk and recent use of dental services. Given the independent association between low fruit and vegetable intake and oral cancer susceptibility, we also evaluated whether long term smokers who consumed fewer than five servings of fruits and vegetables per day, the US National Cancer Institute's recommended intake, were even less likely to visit the dentist than adults who had neither risk factor. Finally, among long term smokers, we explored whether duration of smoking or quantity of cigarettes smoked per day was related to visiting the dentist. We used unconditional logistic regression to estimate odds ratios (ORs) and 95\% confidence intervals (CI). With the exception of income, missing data ranged from $1 \%-3 \%$, and were dropped from the analyses. We excluded income from multivariate analyses because of the substantial amount of missing information, and instead included education, health insurance and dental insurance as proxy measures of this economic access. To account for the survey sampling scheme and weighting of the data, we used SUDAAN software to conduct all analyses. ${ }^{18}$

\section{Results}

A total of 2119 adults age 35 and older met the criteria for inclusion in our study, 30.5\% ( $\mathrm{n}=$ 646) of whom were current smokers or recent former smokers who had smoked for 10 or more years, and $69.5 \%(n=1473)$ of whom were never smokers.

Among long term smokers, $71.1 \%$ had a dental visit in the past year, compared with $83.0 \%$ of never smokers (table 1 ). There was a 


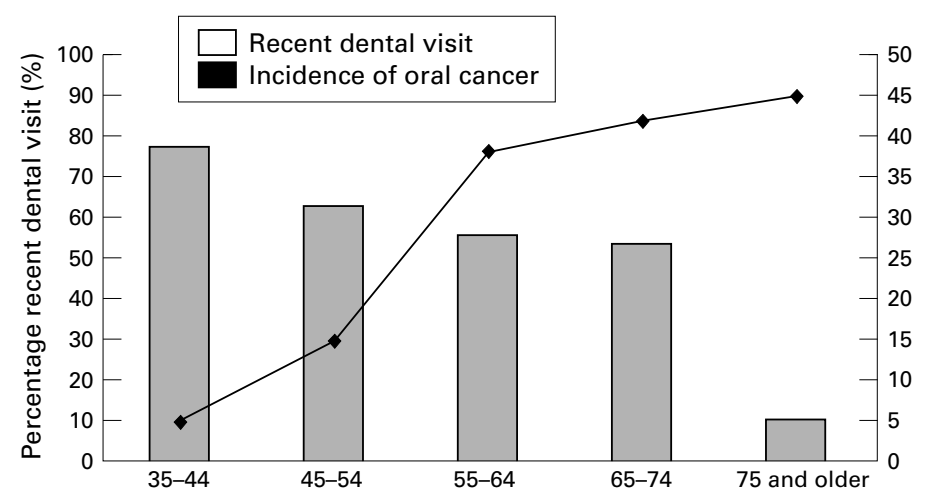

Figure 1 Age specific use of dental services among long term smokers and age specific incidence of oral cancer among Massachusetts adults.

consistent decreased likelihood of a recent dental visit among long term smokers within strata of each demographic or health characteristic. Among both long term and never smokers, higher education and income, and having dental or health insurance were strong predictors of having visited the dentist within the year. Tooth loss also strongly related to whether an adult had recently visited the dentist, and younger age increased the likelihood of a recent dental visit. The effect of age on dental visits was independent of tooth loss (data not shown). The association between age and dental visits is particularly notable among long term smokers, and contrasts with age-specific increases in oral cancer incidence rates (fig 1).

Compared with adults who never smoked cigarettes, long term smokers had only half the odds of visiting the dentist in the previous year (table 2). After adjusting for confounding by demographic and health characteristics, long term smokers remained substantially less likely (adj OR $=0.69,95 \% \mathrm{CI}=0.48,0.99)$ to have had a recent dental visit. When we compared long term smokers with low fruit and vegetable intake $(n=238)$ to adults who had neither risk factor $(n=769)$, we found an even greater disparity in recent dental visits (adj OR $=0.39$,

Table 2 Adjusted ${ }^{\star}$ odds ratios (OR) and 95\% confidence intervals (95\% CI) for the association between oral cancer risk factors and recent dental visits among adults 35 and older

\begin{tabular}{|c|c|c|c|c|}
\hline Preventable risk factor & Number & $\begin{array}{l}\text { Crude } \\
\text { OR }\end{array}$ & $\begin{array}{l}\text { Adjusted } \\
\text { OR }\end{array}$ & $95 \%$ CIt \\
\hline \multicolumn{5}{|l|}{ Smoking } \\
\hline long term smoking & 646 & 0.50 & 0.69 & $0.48,0.99$ \\
\hline never smoker & 1473 & $1 \S$ & $1 \S$ & \\
\hline \multicolumn{5}{|l|}{ Smoking and diet } \\
\hline long term and low fruit/vegetable intake $\ddagger$ & 238 & 0.40 & 0.39 & $0.22,0.68$ \\
\hline never smoker and sufficient fruit/vegetable intake $\ddagger$ & 769 & $1 \Phi$ & $1 \S$ & \\
\hline
\end{tabular}

*Adjusted for gender, age, education, dental insurance, health insurance, recent medical visit, and tooth loss due to decay or gum disease. $\$ 95 \%$ confidence intervals around the adjusted OR. $\ddagger$ Low fruit/vegetable intake equals less than five servings of fruits and vegetables per day and sufficient fruit/vegetable intake equals five+ servings/day. §Reference group.

Table 3 Adjusted ${ }^{\star}$ odds ratios (OR) and 95\% confidence intervals (95\% CI) for the association between smoker characteristics and recent dental visits among long term smokers age 35 and older, Massachusetts BRFSS, 1998

\begin{tabular}{llll}
\hline Smoker characteristics & Crude OR & Adjusted ${ }^{\star}$ OR & 95\% CI \\
\hline $\begin{array}{l}\text { Years smoked } \\
\text { additional 5 years }\end{array}$ & 0.78 & 0.78 & $0.65,0.94$ \\
$\begin{array}{l}\text { Number of cigarettes per day } \\
21 \text { or more (more than 1 pack) } \\
20 \text { or less (a pack or less) }\end{array}$ & 0.52 & 0.55 & $0.28,1.04$ \\
& $1 \neq$ & $1 \neq$ & \\
\hline
\end{tabular}

*Adjusted for gender, age, education, dental insurance, health insurance, and recent medical visit. $\dagger 95 \%$ confidence intervals around the adjusted OR. $\neq$ Reference group.
KEY POINTS

- Early diagnosis of oral cancer involves a simple, low cost examintion of the oral cavity, and early treatment provides good prognosis for patients.

- Survival from oral cancer, however, is among the lowest of all cancer sites, because of the advanced stage at diagnosis.

- Long term smokers, at greater risk of oral cancer, are less likely to have routine dental visits.

- The relation between long term smoking and dental visits is independent of socioeconomic and other health related behaviours.

- Increasing awareness and removing barriers to annual dental visits for long term smokers could reduce mortality and improve overall oral health.

$95 \%$ CI $=0.22,0.68)$, after controlling for potential differences between the groups.

Table 3 shows the association between recent dental vists and smoking duration and quantity among the current, long term smokers. The odds of having a recent dental visit decreased by $22 \%$ (adj OR $=0.78,95 \% \mathrm{CI}=0.65,0.94$ ) with every additional five years that a smoker had smoked, even adjusting for age, tooth loss, and other covariates. In addition, long term smokers who smoked more than a pack of cigarettes per day were $45 \%$ less likely to have recently been to the dentist than adults who smoked a pack or less.

Finally, adults who did not go to the dentist within the year were asked their reasons for not going. Among long term smokers who did not visit the dentist in the past year, $46 \%$ felt that they had no reason to go, while $20 \%$ did not go because of cost, $14 \%$ feared going to the dentist, $7 \%$ said they had other priorities, and $13 \%$ cited other reasons. The pattern of reasons given by never smokers was similar.

\section{Discussion}

Documented at least as far back as the 1930s, advice had been given to the public that "if they are using tobacco, they should have their teeth cleaned at more recent intervals than if they do not smoke". ${ }^{19}$ More recent recommendations support oral cancer examinations as a means to reduce the burden of disease. ${ }^{3}$ While data on the impact on mortality are scant, studies to date show that screening detects precancerous conditions and preclinical malignant lesions with good validity, ${ }^{20}{ }^{21}$ that treatment of precancer reduces the incidence of oral neoplasia, ${ }^{78}$ and that earlier stage disease has better prognosis and reduced morbidity. ${ }^{13}$ Oral cancer screening is also a low cost, non-invasive procedure that is acceptable to the population and takes only two minutes to perform. ${ }^{22}$

Dentists seem the logical providers to perform oral cancer examinations. As part of physical assessments of patients, dentists have a responsibility to perform thorough head and neck examinations. ${ }^{23}$ A survey of dentists in 
Scotland indicated that $94 \%$ include examination of the oral soft tissues as part of usual practice during regular dental check ups. ${ }^{24}$ Among a small sample of Maryland dentists, $78 \%$ stated that they examined most patients for oral cancer on a yearly basis. ${ }^{25} \mathrm{~A}$ population-based study of patients in the United States contradicted these findings, and suggested that only $15 \%$ of adults 40 and older ever received an oral cancer examination. ${ }^{26}$ These data are difficult to interpret, and may in part reflect patients' lack of awareness that a dentist or other provider is conducting an oral cancer examination. ${ }^{27}$

Epidemiological evidence points to a positive association between irregular visits to the dentist and advanced stage of oral cancer at diagnosis. ${ }^{28} 29$ Moreover, Kowalski and colleagues showed both that smokers were more likely to be diagnosed at a later stage of disease than non-smokers, and that there was a dose-response relation between pack years of consumption and increased risk of advanced tumour stage. ${ }^{1}$ We found in our study that long term smokers were less likely to have had a recent dental visit then never smokers, that long term smokers who also consumed a diet low in fruits and vegetables were even less likely to visit the dentist, and that the likelihood of a yearly examination decreased with increasing smoking duration and amount smoked per day. Our data, along with previous research, provide insight into reasons for the substantially low survival rates for oral cancer.

In line with other studies, we found that older adults were less likely to have recently seen a dentist. ${ }^{30}$ The risk of oral cancer greatly increases with age, and older adults are more likely to be diagnosed at advanced stage of disease. $^{3031}$ In this context, the inverse relation between age specific use of dental services among higher risk people and oral cancer incidence (fig 1 ) is striking, and suggests that older higher risk adults are particularly vulnerable to delays in diagnosis.

Cost has often been cited as the principal barrier to dental care for many Americans. ${ }^{30}$ Moreover, the relation between socioeconomic status and smoking is well established. However, our data show that smokers, who are at increased risk for oral cancer, are less likely to visit the dentist even after controlling for socioeconomic status. Strategies that seek to increase accessibility to the dentist by minimising financial barriers may only partially resolve the decreased use of dental services among smokers. Findings from this study may indicate a need for targeted strategies to increase dental visits among smokers.

Several states conducting the BRFSS also included the oral health module in 1998 and additional studies investigating the relation between dental visits and oral cancer risk status would provide further evidence of the generalisability of these findings. We would expect that the overall patterns seen in our study would exist in other states, but that the degree of differential use of dental services might depend upon accessibility of dental services, as well as the health beliefs and status of a given population.

The findings in this study are subject to at least three limitations. Firstly, households without telephones do not have the opportunity to be included in the sample. Overall, $2 \%$ of Massachusetts's households do not have telephones, although $10 \%$ of households living below poverty lack a phone. Secondly, the BRFSS data are based on self report, and are subject to possible reporting biases. Respondents may overreport socially acceptable behaviours, underreport behaviours deemed socially unacceptable, and respondents may have difficulty recalling the frequency or time frame of various behaviours. Thirdly, the response rate to the BRFSS has been decreasing over time, and in 1998 was $59 \%$. If the relation between long term smoking and recent dental visits was different for people who did and did not respond to the survey, a bias in our analysis could have occurred.

While dentists may be the logical choice to perform screening, our data strongly support a role for other health professionals to conduct examinations of the oral cavity. Almost $88 \%$ of at risk adults visited either a dentist or a physician within the past year, and older adults were much more likely to have had a routine medical visit than dental visit. In a study by Prout and colleagues, oral cancer patients often had multiple medical visits in the months before their diagnosis, while no intraoral examination was done. ${ }^{32}$ Currently, the percentage of non-dental health professionals who perform oral cancer screening is much lower than dentists. ${ }^{27}$ Programmes targeted to all health professionals to increase education and awareness about intraoral exams could certainly contribute to detecting oral cancer at an earlier stage.

Use of tobacco increases the risk of several oral conditions, including tooth loss, periodontal disease, and oral soft tissue lesions. ${ }^{33}{ }^{34}$ The findings from this study have implications beyond the scope of oral cancer, and suggest that adults at higher risk for a variety of oral conditions are less likely to regularly visit the dentist. Untreated disease can increase the severity of conditions, contribute to significant damage to the oral cavity, necessitate costly treatment, and may ultimately raise the risk of other systemic conditions. ${ }^{35-37}$ Increasing the percentage of smokers who visit the dentist annually and/or receive intraoral exams from other health professionals should improve oral health in a population at greatest risk of disease.

The authors would like to express their gratitude to the residents of Massachusetts who participated in this survey, and to thank Dr Chester Douglass for his helpful comments on this manuscript.

Funding: this research was supported by the Massachusetts Department of Public Health and by the Centers for Disease Control and Prevention via Cooperative Agreement U58/ CCU115077-01.

Conflicts of interest: none. Kowalski LP, Franco EL, Torloni H, et al. Lateness of diag-
nosis of oral and oropharyngeal carcinoma: factors related
to the tumour, the patient and health professionals. Eur $\mathcal{F}$ Cancer B Oral Oncol 1994;30B:167-73.
Co the tumour, the patient and health 
2 National Cancer Institute SEER Program. SEER cancer statistics review 1973-1996.

3 American Cancer Society. 1999 cancer facts and figures. Atlanta, GA: Publication 99-300M-no, 5008.99.

4 Daftary DK, Murti PR, Bhonsle RR, et al. Risk factors and risk markers for oral cancer in high risk areas of the world In: Johnson NW, ed. Oral cancer: the detection of patients and lesions at risk. Cambridge: Cambridge University Press, 1991:29-63.

5 Gupta PC, Bhonsle RB, Murti PR, et al. An Epidemiologic assessment of cancer risk in oral precancerous lesions in India with special reference to nodular leukoplakia. Cancer 1989;63:2247-52.

6 Schepman KP, van der Maij EH, Smeele LE, et al. Malignant transformation of a hospital-based population of 166 patients with oral leukoplakia from the Netherlands. Oral Oncol 1998;34:270-5.

7 Hong WK, Lippman SM, Itri LM, et al. Prevention of secondary tumours with isotretinoin in squamous-cell carcinoma of the head and neck. N Engl F Med 1990;323:795801.

8 Gupta PC, Mehta FS, Pindborg JJ, et al. Primary prevention trial of oral cancer in India: a 10-year follow-up study. $\mathcal{f}$ Oral Pathol Med 1992;21:433-9.

9 Franceschi S, Barzan L, Talamini R. Screening for cancer of the head and neck: if not now, when? Oral Oncol 1997;33: 313-16.

10 Malloawalla AM, Silverman S, Mani NJ, et al. Oral cancer in 57,581 industrial workers in Gujrat India. A prevalence in 57, 581 industrial workers in Gujrat India.
and follow-up study. Cancer 1976;37:1882-6.

11 Macfarlane GJ, Zheng T, Marshall JR, et al. Alcohol, tobacco, diet and the risk of oral cancer: a pooled analysis of three case-control studies. Eur $\mathcal{F}$ Cancer B Oral Oncol 1995;31B: $181-7$

12 Rothman KJ. Epidemiology of head and neck cancer. Laryngoscope 1978;88:435-8.

13 Graham S. Dentition, diet, tobacco and alcohol in the epidemiology of oral cancer. F Natl Cancer Inst 1977;59. 1611-18.

14 Franco EL, Kowlaski LP, Oliveria BV, et al. Risk factors for oral cancer in Brazil: a case-control study. Int $\mathcal{f}$ Cancer 1989;43:992-1000.

15 Francheschi S, Talamini R, Barra S, et al. Smoking and drinking in relation to cancers of the oral cavity, pharynx, drinking in relation to cancers of the oral cavity, pharynx, larynx, and $50: 6502-7$.

16 Powell-Griner E, Anderson JE, Murphy W. State and sex-specific prevalence of selected characteristicsBehavioral Risk Factor Surveillance System, 1994 and 1995. In: CDC Surveillance Summaries, August 1, 1997. MMWR 1997;46 (no SS-3):1-31.

17 Owen N and Brown SL. Smokers unlikely to quit. $\mathcal{F}$ Behav Med 1991;14:627-6.

18 Shah BV, Barnwell BV, Bieler GS. SUDAAN users manual and software, release 7.0. Research Triangle Park, NC: Research Triangle Institute, 1996.
19 Quinn ME, Dudley A. Where there's smoke, there's fire: a survey of smoking conducted at Massachusetts Clinics, 1932 and Health, 1952 .

20 Jullien JA, Downer MC, Zakrzewska JM, et al. Evaluation of a screening test for the early detection of oral cancer and precancer. Community Dent Health 1995;12:3-7.

21 Downer MC, Evans AW, Hughes Hallett CM, et al. Evaluation of screening for oral cancer and precancer in a company headquarters. Community Dent Oral Epidemiol 1995;23:302-5.

22 Niessen LC, Jones JA, Lonergan JJ. Oral examination of the geriatric patient. Geriatr Med Today 1986;5:113-16.

23 Fedele DJ, Jones JA, Niessen LC. Oral cancer screening in the elderly. 7 Am Geriatr Soc 1991;39:920-25.

24 Cowan CG, Gregg TA, Kee F. Prevention and detection of oral cancer: the views of primary care dentists in Northern Ireland. Br Dent f 1995;179:338-42.

25 Yellowitz JA, Goodman HS. Assessing physicians' and dentists' oral cancer knowledge, opinions, and practices. $7 \mathrm{Am}$ Dent Assoc 1995;126:53-60.

26 Horowitz AM, Nourjah PA. Factors associated with having an oral cancer examination among US adults 40 years of age and older. F Public Health Dent 1996;56:331-5.

27 Martin LM, Bouquot JE, Wingo PA, et al. Cancer prevention in the dental practice: oral cancer screening and tobacco cessation advice. F Public Health Dent 1996;56:336-40.

28 Rubright WC, Hoffman HT, Lynch CF, et al. Risk factors for advanced stage oral cavity cancer. Arch Otolaryngol Head Neck Surg 1996;122:621-6.

29 Elwood JM, Gallagher RP. Factors influencing early diagnosis of cancer of the oral cavity Can Med Assoc 7 1985;133:651-6.

30 Oral Health Coordinating Committee, Public Health Service. Toward improving the oral health of Americans: an overview of oral health status, resources, and care delivery. Public Health Rep 1993;108:657-72.

31 Oral cancer: incidence by age and stage at diagnosis. SEER Registries, 1992-1996. Data from SEER ${ }^{\star}$ State, $4 / 99$ ed.

32 Prout MN, Heeren TC, Barber CE, et al. Use of health services before diagnosis of head and neck cancer among Boston residents. Am f Prev Med 1990;6:77-83.

33 Krall EA, Garvey AJ, Garcia RI. Alveolar bone loss and tooth loss in male cigar and pipe smokers. Am Dent Assoc 1999;130:57-64

34 Machtei EE, Hausmann E, Dunford R, et al. Longitudinal study of predictive factors for periodontal disease and tooth loss. F Clin Periodontol 1999;26:374-80.

35 Beck JD, Offenbacher S. Oral health and systemic disease: periodontitis and cardiovascular disease. F Dent Educ 1998; 62:859-70.

36 Joshipura KJ, Rimm EB, Douglass CW, et al. Poor oral health and coronary heart disease. F Dent Res 1996;75: 1631-6.

37 Offenbacher S, Katz V, Fertik G, et al. Periodontal infection as a possible risk factor for preterm low birth weight. F Periodontol 1996;67 (suppl):1103-13. 